\title{
Pathophysiology of Obstructive Sleep Apnea: The Role of Physiologic Phenotypes
}

\author{
Alexandre R Abreu ${ }^{1}$, Alberto R Ramos ${ }^{2}$, Salim I Dib ${ }^{3}$, Carlos Torre ${ }^{4}$, Alejandro D Chediak ${ }^{5}$
}

\begin{abstract}
Obstructive sleep apnea (OSA) is a common medical disorder of increasing interest in the medical community because of evidence that significant cardiovascular and neurocognitive defects occur when OSA is untreated or inadequately treated. Positive airway pressure (PAP) therapy remains the most effective and widely prescribed treatment for OSA. However, long-term adherence to PAP therapy has proven challenging. Alternative surgical and medical therapies are available, but an estimation of treatment efficacy based on clinical grounds has yielded inconsistent and largely disappointing results. The identification of a unique mechanism for OSA in a given clinical subject should prove useful in the selection of treatment alternatives to PAP. Until recently, personalizing treatment of OSA based on the determination of the mechanism of action was available only in experimental settings requiring invasive instrumentation. However, it is now possible to analyze clinical polysomnography data and identify the physiologic mechanism(s) or phenotype(s) of OSA in an individual case. The determination of physiologic phenotype offers the opportunity for personalized therapy. Additionally, physiologic phenotyping of OSA provides an opportunity to understand OSA mechanisms in specific subgroups, such as the elderly and the obese without OSA. In this manuscript, we introduce the reader to the concept and techniques of physiologic phenotyping of OSA and summarize the scientific data that physiologic phenotyping confers to understanding and treating OSA. Keywords: Loop gain, Obstructive sleep apnea, Pharyngeal critical closing pressure, Phenotypes, Respiratory arousal responses, Sleep apnea, Upper airway control.

International Journal of Head and Neck Surgery (2021): 10.5005/jp-journals-10001-1428
\end{abstract}

\section{INTRODUCTION}

Obstructive sleep apnea (OSA) is a common disorder widely recognized because of increasing prevalence and compelling evidence demonstrating significant neurocognitive and cardiovascular sequela of untreated OSA. ${ }^{1-3}$ The condition is characterized by repetitive sleep-related narrowing or closure of the upper airway with associated hypoxia and sleep fragmentation. These obstructive events are termed apneas and hypopnea. The frequency of apnea and hypopnea per hour of sleep, the apneahypopnea index $(\mathrm{AHI})$, is the most widely accepted metric of sleep apnea presence and severity. ${ }^{4}$

Positive airway pressure (PAP) therapy is efficacious therapy for OSA, yet many patients are PAP intolerant and others only partially adherent to PAP therapy. ${ }^{5}$ Understanding the pathogenesis of OSA and a given individual would be useful to select personalized therapy.

Narrowing of the upper airway, either by obesity or anatomic factors, has traditionally been implicated as the primary disturbance driving the pathogenesis of OSA. However, OSA exists in the absence of either obesity or clinically apparent structural compromise of the upper airway. Hence, it follows that the pathogenesis of OSA varies among individuals. ${ }^{6-8}$ Identifying specific pathogenic mechanisms can be used to individualize the treatment of OSA. In this manuscript, we will review the pathophysiologic mechanisms of OSA with the aim to personalize treatment paradigms targeting the underlying physiologic derangement leading to upper airway compromise during sleep. ${ }^{9}$

\section{Physiologic Mechanisms of OSA}

Physiologic studies of upper airway function in OSA have identified several mechanisms of sleep-related upper airway instability. Key
1,5 Division of Pulmonary, Critical Care and Sleep Medicine, Department of Medicine, University of Miami, Miller School of Medicine, Miami, Florida, USA

${ }^{2,3}$ Department of Neurology, University of Miami, Miller School of Medicine, Miami, Florida, USA

${ }^{4}$ Department of Otolaryngology-Head and Neck Surgery, University of Miami, Miller School of Medicine, Miami, Florida, USA

Corresponding Author: Alejandro D Chediak, Division of Pulmonary, Critical Care and Sleep Medicine, Department of Medicine, University of Miami, Miller School of Medicine, Miami, Florida, USA, Phone: +1 305-243-9999, e-mail: axc1599@med.miami.edu

How to cite this article: Abreu AR, Ramos AR, Dib $\mathrm{Sl}$, et al. Pathophysiology of Obstructive Sleep Apnea: The Role of Physiologic Phenotypes. Int J Head Neck Surg 2021;12(2):71-73.

Source of support: Nil

Conflict of interest: None

mechanisms underlying sleep-induced upper airway narrowing and occlusion include: ${ }^{7}$

- Anatomic compromise or collapsibility of the upper airway is often assessed by the critical pharyngeal closing pressure (Pcrit).

- Inadequate responsiveness of upper airway dilator muscles during sleep determined by the low upper airway electromyogram (EMG) response to negative luminal pressure.

- Low respiratory arousal threshold defined as the level of respiratory drive that causes arousal.

- Hyperresponsiveness of the ventilatory control system or increased respiratory loop gain.

Reducing Pcrit, increasing EMG responsiveness of the genioglossus, increasing arousal threshold, or lowering loop

(c) The Author(s). 2021 Open Access This article is distributed under the terms of the Creative Commons Attribution 4.0 International License (https:// creativecommons.org/licenses/by-nc/4.0/), which permits unrestricted use, distribution, and non-commercial reproduction in any medium, provided you give appropriate credit to the original author(s) and the source, provide a link to the Creative Commons license, and indicate if changes were made. The Creative Commons Public Domain Dedication waiver (http://creativecommons.org/publicdomain/zero/1.0/) applies to the data made available in this article, unless otherwise stated. 
gain has been shown to reduce the frequency of OSA events. Interventions targeting underlying pathophysiology are expected to be beneficial if individualized. Uvulopalatopharyngoplasty may prove most beneficial for patients with an anatomic compromise of the velopharynx and stability of the respiratory controls center. ${ }^{10}$ Raising hypoglossal nerve output should be most beneficial for those patients with impaired upper airway motor responsiveness while efforts to stabilize respiratory control benefit patients with increased loop gain as the cause for sleep apnea. ${ }^{11}$ Finally, low arousal threshold phenotypes arouse prematurely and have inadequate time for accumulation of respiratory drive sufficient to activate pharyngeal dilator muscles and stabilize the upper airway. Increasing the arousal threshold with pharmaceuticals improves $\mathrm{AHI}$ in low arousal phenotype by allowing time for activation of upper airway muscles to reestablish upper airway patency. Table 1 describes some possible interventions specific to unique physiologic phenotypes of OSA. Some with OSA may have more than one perturbation thereby requiring combined therapy.

\section{Determining Physiologic Phenotypes of OSA Using Clinical Polysomnography}

Until recently, a significant limitation of the phenotype/personalized approach to managing sleep apnea has been the need for complex and invasive overnight testing to identify the underlying mechanism in each case. Clinicians now have available the tools necessary to assess control of breathing, arousal threshold upper airway collapsibility, and muscle responsiveness via clinical sleep recordings. The lines that follow briefly describe and reference the available methods to determine a specific physiologic trait.

\section{Determining Upper Airway Collapsibility and Muscle Responsiveness}

Pharyngeal collapsibility (Pcrit) can be defined physiologically as the ventilation at eupneic respiratory drive during sleep. Pharyngeal compensation (muscle responsiveness) to spontaneously occurring obstructive upper airway events can be measured as the ventilation accompanying increasing respiratory drive during respiratory events. In patients with OSA, Sands et al. ${ }^{12}$ used a novel automated noninvasive method to estimate ventilatory drive during polysomnography and compared that to ventilatory drive measured using the gold standard invasive method with an intraesophageal diaphragm EMG and measures using an abrupt drop in CPAP to cause airway narrowing. Polysomnography derived collapsibility and compensation estimates correlated favorably with those derived using ventilatory drive techniques $(R=0.83$, $p<0.0001$; and $R=0.76, p<0.0001$; respectively) and with metrics

Table 1: Personalized treatment of OSA based on physiologic phenotype

- Anatomic compromise with stable respiratory control

- Surgical intervention

- Inadequate responsiveness of upper airway muscles

- Electrical stimulation of the upper airway

- Pharmaceutical stimulation of the upper airway

- Low respiratory arousal threshold

- Prescription hypnotics (eszopiclone)

- Increased loop gain

- Supplemental oxygen

- Acetazolamide made using CPAP drops allowing airway narrowing $(R=0.67, p<$ 0.0001 ; and $R=0.64, p<0.001$; respectively). The data indicate that phenotypes of pharyngeal dysfunction in OSA can be assessed from spontaneous changes in ventilation and ventilatory drive during sleep using data derived during clinical polysomnography.

\section{Determination of Arousal Threshold}

Using anthropometric data and metrics derived clinical polysomnography, Edwards et al. ${ }^{13}$ showed that arousal threshold can be reliably estimated from the $\mathrm{AHI}$, nadir oxyhemoglobin saturation, and the fraction of hypopnea relative to apnea events. They developed a clinical score (Table 2 ) that correctly predicted low arousal threshold in $84.1 \%$ of participants with a sensitivity of $80.4 \%$ and specificity of $88 \%$.

\section{Determination of Loop Gain}

Loop gain, as it pertains to the respiratory system, can be defined as the ventilatory response to a given ventilatory disturbance (response/disturbance). Terrill et al. ${ }^{14}$ described fitting a ventilatory control model of the ventilatory drive to the ventilatory pattern of OSA reveals the underlying loop gain. The model was used to quantify loop gain from diagnostic polysomnography vs loop gain derived by the CPAP drop method. Additionally, they assessed the accuracy of the model in detecting the expected reductions in loop gain that occurs with supplemental oxygen and acetazolamide. Correlation vs the CPAP drop technique was $0.63, p<0.001$. Similarly, the model predicted the OSA response to loop gain lowering therapy with oxygen and acetazolamide.

\section{Insight Gained from OSA Phenotyping}

Physiologic phenotyping of patients with OSA may prove useful in personalized treatment selection and can be used to elucidate the pathogenesis of OSA in specific OSA subgroups. Large body habitus is recognized as a major determinant of OSA. However, many individuals do not have OSA despite being overweight or obese. Using a CPAP drop technique, investigators have shown enhanced upper airway muscle responsiveness as a characteristic feature of overweight/obese individuals without OSA. ${ }^{8}$ One study comparing physiologic phenotypes of young (age 20-40 years) to old (age $\geq 60$ years) subjects with OSA suggested that airway anatomy/ collapsibility plays a relatively greater pathogenic role in older adults while a sensitive ventilatory control system (higher loop gain) is a more prominent trait in younger adults with OSA. ${ }^{6}$ Increasing arousal threshold with the prescription benzodiazepine receptor agonist hypnotic eszopiclone can reduce $\mathrm{AHI}$ in OSA patients with low arousal threshold phenotype but not in OSA patients with other physiologic phenotypes. ${ }^{15}$ Finally, Joosten and company ${ }^{10}$ recently showed that upper airway surgery is most effective in OSA patients

Table 2: Determining low arousal threshold from routine diagnostic polysomnography reports ${ }^{13}$

- Assign a value of 1 for each of the following criteria:

(a) $\mathrm{AHI}<30$

(b) Fraction of hypopnea to apnea $>58.3 \%$

- (\#hypopneas/\#apneas + \#hypopneas) $\times 100$

(c) Oxyhemoglobin saturation nadir $>82.5 \%$

- Sum $a+b+c$ above

- Sum $\geq 2$ meets criteria for low arousal threshold 
with lower loop gain, an observation that can be used clinically to pre-select patients against surgical failure.

\section{Future Directions}

Recent studies have identified mechanisms by which clinical polysomnography can be used to determine physiologic phenotypes. Certainly, determination of low arousal threshold phenotype can be readily accomplished for measures routinely reported during diagnostic polysomnography (Table 1). However, the estimation of upper airway collapsibility, upper airway muscle responsiveness, and loop gain require sophisticated computation software and techniques that are beyond the capabilities of all but a few clinical sleep laboratories. Further, the techniques have been validated in small groups of subjects by a limited group of investigators. Hence, physiologic phenotyping of OSA with clinical polysomnography, while promising to facilitate widespread adoption and, consequently, personalized treatment of OSA, remains just beyond the reach of most clinicians. Standardizing of data collection protocols and simplification of ventilatory modeling are necessary for physiologic phenotyping to be a routine component of clinical polysomnography. Finally, longterm studies are necessary to demonstrate that personalized treatment of OSA based on physiologic phenotypes improves healthcare outcomes.

All authors met the criteria for authorship established by the International Committee of Medical Journal Editors: Alexandre R Abreu, MD and Alejandro D Chediak, MD, were responsible for the conception, design, and drafting of the work, revising the work, and reviewing the manuscript. Alberto R Ramos MD, Carlos Torre MD, and Salim I Dib MD, offered substantial contributions in drafting, revising, and reviewing the manuscript. All authors provided final approval of the version to be published and agreed to be accountable for all ensuing aspects of the work.

\section{References}

1. Peppard PE, Young T, Barnet JH, et al. Increased prevalence of sleepdisordered breathing in adults. Am J Epidemiol 2013;177(9):10061014. DOI: 10.1093/aje/kws342.

2. Kapur V, Blough DK, Sandblom RE, et al. The medical cost of undiagnosed sleep apnea. Sleep 1999;22(6):749-755. DOI: 10.1093/ sleep/22.6.749.
3. Lam JC, Mak JC, Ip MS. Obesity, obstructive sleep apnoea and metabolic syndrome. Respirology (Carlton, Vic) 2012;17(2):223-236. DOI: 10.1111/j.1440-1843.2011.02081.x.

4. Berry R, Brooks R, Gamaldo C, et al. In: Medicine AAoS, ed., The AASM manual for the scoring of sleep and associated events: rules, terminology and technical specifications. Darien, Illinois: American Academy of Sleep Medicine; 2017.

5. Weaver TE, Grunstein RR. Adherence to continuous positive airway pressure therapy: the challenge to effective treatment. Proceed Am Thora Soc 2008;5(2):173-178. DOI: 10.1513/pats.200708-119MG.

6. Edwards BA, Wellman A, Sands SA, et al. Obstructive sleep apnea in older adults is a distinctly different physiological phenotype. Sleep 2014;37(7):1227-1236. DOI: 10.5665/sleep.3844.

7. Younes $M$, Ostrowski $M$, Atkar R, et al. Mechanisms of breathing instability in patients with obstructive sleep apnea. J Appl Physiol 2007;103(6):1929-1941. DOI: 10.1152/japplphysiol.00561.2007.

8. Sands SA, Eckert DJ, Jordan AS, et al. Enhanced upper-airway muscle responsiveness is a distinct feature of overweight/obese individuals without sleep apnea. Am J Respirat Criti Care Med 2014;190(8):930937. DOI: $10.1164 / \mathrm{rccm} .201404-07830 \mathrm{C}$.

9. Eckert DJ, White DP, Jordan AS, et al. Defining phenotypic causes of obstructive sleep apnea. Identification of novel therapeutic targets. Am J Respirat Criti Care Med 2013;188(8):996-1004. DOI: 10.1164/ rccm.201303-0448OC.

10. Joosten SA, Leong $\mathrm{P}$, Landry SA, et al. Loop gain predicts the response to upper airway surgery in patients with obstructive sleep apnea. Sleep 2017;40(7). DOI: 10.1093/sleep/zsx094.

11. Edwards BA, Sands SA, Eckert DJ, et al. Acetazolamide improves loop gain but not the other physiological traits causing obstructive sleep apnoea. J Physiol 2012;590(5):1199-1211. DOI: 10.1113/ jphysiol.2011.223925.

12. Sands SA, Edwards BA, Terrill PI, et al. Phenotyping pharyngeal pathophysiology using polysomnography in patients with obstructive sleep apnea. Am J Respirat Criti Care Med 2018;197(9):1187-1197. DOI: 10.1164/rccm.201707-1435OC.

13. Edwards BA, Eckert DJ, McSharry DG, et al. Clinical predictors of the respiratory arousal threshold in patients with obstructive sleep apnea. Am J Respirat Criti Care Med 2014;190(11):1293-1300. DOI: 10.1164/rccm.201404-07180C.

14. Terrill PI, Edwards BA, Nemati S, et al. Quantifying the ventilatory control contribution to sleep apnoea using polysomnography. The Eur Respirat J 2015;45(2):408-418. DOI: 10.1183/09031936.00062914.

15. Eckert DJ, Owens RL, Kehlmann GB, et al. Eszopiclone increases the respiratory arousal threshold and lowers the apnoea/hypopnoea index in obstructive sleep apnoea patients with a low arousal threshold. Clin Sci (Lond) 2011;120(12):505-514. DOI: 10.1042/ CS20100588. 